\title{
Avaliação da modulação autonômica mediante estímulo musical em indivíduos musicalizados e não musicalizados
}

\author{
Evaluation of autonomic modulation through musical stimulation in musicalized and non- \\ musicalized individuals
}

Evaluación de la modulación autonómica mediante estimulación musical en individuos musicalizados y no musicalizados

Fernando Seiji da Silva ORCID: https://orcid.org/0000-0002-6373-8817 Universidade Federal do Triângulo Mineiro, Brasil E-mail: fernando.silva@uftm.edu.br

Luciana Duarte Novais Silva ORCID: https://orcid.org/0000-0002-1200-5680 Universidade Federal do Triângulo Mineiro, Brasil E-mail: luciana.duarte.silva@uftm.edu.br

Solon Quintão Henriques Júnior ORCID: https://orcid.org/0000-0003-1804-6341 Universidade Federal do Triângulo Mineiro, Brasil E-mail: solonquintao@gmail.com

Jairo da Silva Custódio Júnior ORCID: https://orcid.org/0000-0002-4572-2117 Universidade Federal do Triângulo Mineiro, Brasil E-mail: jairoscjunior@gmail.com

Ana Paula Espindula

ORCID: https://orcid.org/0000-0002-9282-4482 Universidade Federal do Triângulo Mineiro, Brasil E-mail: ana.espindula@uftm.edu

Leonardo Augusto Lombardi ORCID: https://orcid.org/0000-0002-9735-8804 Universidade Federal do Triângulo Mineiro, Brasil E-mail: nda_leo@yahoo.com.br

Ricardo Luiz Smith

ORCID: https://orcid.org/0000-0001-8103-7371 Universidade Federal de São Paulo, Brasil E-mail: rlsmith@unifesp.br

\begin{abstract}
Resumo
O objetivo foi avaliar a modulação autonômica da frequência cardíaca em estímulos musicais em indivíduos musicalizados (GM) e não musicalizados (GNM). Foram avaliados 96 voluntários. Quatro músicas diferentes (M1, M2, M3 e M4) foram executadas. A análise dos dados foi feita no domínio do tempo pelos índices de RMSSD (raiz quadrada da média do quadrado das diferenças entre intervalos da variabilidade da frequência cardíaca consecutivos. Este parâmetro fornece uma quantificação das variações abruptas da variabilidade) e pNN50 (NN50: quantidade absoluta (contagem) de intervalos $\mathrm{NN}$ que diferem mais de 50ms em relação ao intervalo anterior - pNN50: porcentagem de NN50 em relação à quantidade total de intervalos NN), e no domínio da frequência pelos índices de baixa frequência - LF (atividade simpática), alta frequência - HF (atividade parassimpática) e LF/HF (balanço simpático-vagal). Resultados: O GM apresentou menor atividade parassimpática durante a exposição a diferentes tipos de música, independente do sexo $(\mathrm{p}<0,05)$. Porém, durante a execução da $\mathrm{M} 3$, foram observados valores maiores de BF no grupo feminino $(p<0,05)$. No grupo masculino foram encontrados aumento na banda $\mathrm{BF}$ e na relação $\mathrm{BF} / \mathrm{AF}$ durante a execução de música excitatória e sedativa $(\mathrm{p}<0,05)$. No $\mathrm{GNM}$, os resultados foram semelhantes entre os diferentes tipos de música, confirmados por comparação específica entre grupos onde houve diferenças significativas entre eles ( $\mathrm{p}<0,05)$, exceto no BF durante o M3, com valores menores para o GNM. Concluise que o estímulo músical provocou alteração da VFC nos individuos avaliados.
\end{abstract}

Palavras-chave: Modulação autonômica; Frequência cardíaca; Música.

\section{Abstract}

The objective was to evaluate the autonomic modulation of heart rate in musical stimuli in musicalized (GM) and non-musicalized (GNM) individuals. 96 volunteers were evaluated. Four different songs (M1, M2, M3 and M4) were 
performed. Data analysis was performed in the time domain using RMSSD (square root of the mean square of the differences between consecutive heart rate variability intervals) and pNN50 (NN50: absolute quantity) indices. (count) of NN intervals that differ by more than $50 \mathrm{~ms}$ from the previous interval - pNN50: percentage of NN50 in relation to the total amount of NN intervals), and in the frequency domain by low frequency indices - LF (sympathetic activity), high frequency - HF (parasympathetic activity) and LF/HF (sympathetic-vagal balance). Results: GM showed lower parasympathetic activity during exposure to different types of music, regardless of gender $(\mathrm{p}<0.05)$. However, during the execution of M3, higher BF values were observed in the female group $(\mathrm{p}<0.05)$. In the male group, an increase in the $\mathrm{BF}$ band and in the $\mathrm{BF} / \mathrm{AF}$ ratio was found during the performance of excitatory and sedative music $(\mathrm{p}<0.05)$. In GNM, the results were similar between different types of music, confirmed by specific comparison between groups where there were significant differences between them $(\mathrm{p}<0.05)$, except in BF during M3, with lower values for GNM. It is concluded that the musical stimulus caused changes in the HRV in the individuals evaluated.

Keywords: Autonomic modulation; Heart rate; Song.

\section{Resumen}

El objetivo fue evaluar la modulación autónoma de la frecuencia cardíaca en estímulos musicales en individuos musicalizados (GM) y no musicalizados (GNM). Se evaluaron 96 voluntarios. Se interpretaron cuatro canciones diferentes (M1, M2, M3 y M4). El análisis de datos se realizó en el dominio del tiempo utilizando los índices RMSSD (raíz cuadrada del cuadrado medio de las diferencias entre intervalos consecutivos de variabilidad de la frecuencia cardíaca) y pNN50 (NN50: cantidad absoluta). (recuento) de intervalos NN que difieren en más de $50 \mathrm{~ms}$ del intervalo anterior - pNN50: porcentaje de NN50 en relación con la cantidad total de intervalos NN), y en el dominio de frecuencia por índices de frecuencia baja - LF (actividad simpática), alta frecuencia - HF (actividad parasimpática) y LF / HF (equilibrio simpático-vagal). Resultados: GM mostró menor actividad parasimpática durante la exposición a diferentes tipos de música, independientemente del género $(\mathrm{p}<0.05)$. Sin embargo, durante la ejecución de M3, se observaron valores más altos de BF en el grupo de mujeres ( $\mathrm{p}<0.05$ ). En el grupo masculino, se encontró un aumento en la banda BF y en la relación BF / AF durante la ejecución de música excitadora y sedante ( $\mathrm{p}<0.05)$. En GNM, los resultados fueron similares entre diferentes tipos de música, confirmado por la comparación específica entre grupos donde hubo diferencias significativas entre ellos ( $\mathrm{p}<0.05$ ), excepto en $\mathrm{BF}$ durante M3, con valores más bajos para GNM. Se concluye que el estímulo musical provocó cambios en la VFC en los individuos evaluados.

Palabras clave: Modulación autónoma; Ritmo cardíaco; Canción.

\section{Introdução}

O som é captado pelo sistema auditivo, que por sua vez é formado por três componentes: condutivo (composto pela orelha externa e média), sensorial (a cóclea) que transforma o impulso sonoro em elétrico, e o componente neural. Os dois primeiros componentes, condutivo e sensorial, fazem parte do sistema periférico, e o componente neural, responsável pela atividade central, faz parte do sistema nervoso. A atividade periférica realiza a detecção e transmissão dos sons, e a atividade central faz a discriminação, localização, reconhecimento do som, compreensão, atenção seletiva e memória auditiva. As estruturas do sistema nervoso central que compõem o sistema auditivo são: o tronco encefálico, vias subcorticais, córtex auditivo, lobo temporal e corpo caloso. Sendo possível associar determinadas funções a algumas estruturas do sistema auditivo central (Steiner, 1999).

A música é o som que é organizado segundo os princípios do ritmo, altura, harmonia e que emprega timbres musicais que nos permitem diferenciar fontes de som e identificar instrumentos musicais (Limb, 2006). A música existe na sociedade humana desde o período da pré-história, provavelmente devido à possibilidade de se expressar, da regulação das emoções e pelo prazer proporcionado ao ouvi-la (Zatorre, Salimpoor, 2013). Estudos clínicos indicam que a música diminui a ansiedade, reduz a pressão arterial e promove alteração na variabilidade da frequência cardíaca (Nakamura et al., 2007; Nogueira et al., 2015; Nieto-Romero, 2017; Melo et al., 2018; Tshiswaka \& Pinheiro, 2020; Silva et al., 2021; Seiji et al., 2021) e a frequência cardíaca em situações estressantes associadas a procedimentos da clínica laboratorial (Sokhadze, 2007), sendo utilizada inclusive para tratamentos de desordens neuropsiquiátricas (Fukui \& Toyoshima, 2008).

Para o cérebro humano uma das fontes mais poderosas de estimulação auditiva é provida pela música. Escutá-la é um processo complexo para o cérebro, já que aciona várias sequências de substratos distintos tanto cognitivos quanto emocionais (Peretz, 2002; Limb, 2006). Urakawa e Yokoyama (2005) demonstraram que exercícios físicos ativam uma dominância simpática do SNA (sistema nervoso autônomo), e que se o exercício físico for acompanhado de música isso acentuaria essa 
dominância simpática, promovendo uma excitação fisiológica, aumentando, consequentemente, a atividade fisiológica, além de tornar a realização de tal atividade mais prazerosa. A música também é capaz de gerar alterações autonômicas mesmo em indivíduos em estado vegetativo, como demonstrado por Riganello et al. (2010). Tais alterações podem ter possíveis valores emocionais, o que implicaria em uma resposta residual nestes indivíduos a estímulos complexos.

Uma forma não invasiva para se avaliar a modulação autonômica da frequência cardíaca é por meio da variabilidade da frequência cardíaca (VFC), que avalia as oscilações periódicas da frequência cardíaca instantânea (batimento a batimento cardíaco) e da duração dos intervalos R-R do eletrocardiograma. Essas oscilações estão na dependência da atividade do sistema nervoso simpático e parassimpático (Catai et al., 2002). As fibras nervosas eferentes do SNA, cujas especificidades de controle são distintas, promovem estimulação ou inibição do coração, tendo influência tônica e reflexa sobre as principais variáveis do sistema cardiovascular (pressão arterial, resistência periférica e débito cardíaco). A liberação de noradrenalina no coração modifica o débito cardíaco por modificar a força de contração das fibras do miocárdio e a frequência cardíaca (FC), enquanto a liberação de noradrenalina na parede dos vasos sanguíneos de resistência da circulação sistêmica altera o estado contrátil da musculatura lisa vascular e, por conseguinte, a resistência vascular periférica (Franchini, 1998).

Em relação às vias eferentes de conexão entre o sistema nervoso central e o controle do sistema cardiovascular, sabese que o sistema nervoso parassimpático, por intermédio dos nervos vagos, originários na base do crânio, carreiam impulsos para os nódulos sinoatrial e atrioventricular, para os átrios e para os ventrículos. Tendo como neurotransmissor a acetilcolina, o vago provoca um efeito cronotrópico negativo, diminuindo a FC (Blair et al., 1988). Já o sistema nervoso simpático exerce influência sobre todo o aparelho circulatório, por meio dos nervos simpáticos originários do tronco cervical e torácico superior. Libera noradrenalina como neurotransmissor e estimula o coração, provocando aumento da $\mathrm{FC}$ e aumento da força de contração (Blair et al., 1988).

Segundo a European Society of Cardiology e the North American Society of Pacing Electrophysiology (1996), a VFC é uma das mais confiáveis e acessíveis fontes de informação sobre a modulação do sistema nervoso autônomo sobre o sistema cardiovascular e pode ser estudada em repouso nas posições supina e sentada, durante sono e, ou vigília, durante exercício físico, durante exposição à música, dentre uma série de outras condições (Silva et al., 2001; Catai et al., 2002; Nakahara al et., 2011). Dentre os métodos para se avaliar a VFC, o mais simples é a medida no domínio do tempo (DT), onde a FC em qualquer ponto no tempo ou iR-R correspondentes são determinados. Tal medida baseia-se em cálculos estatísticos simples realizados nas séries de iR-R (European Society of Cardiology e the North American Society of Pacing Electrophysiology, 1996). Outra forma é a análise no domínio da frequência (DF), que, por meio da análise espectral, decompõe a VFC em componentes oscilatórios fundamentais, sendo que os principais são: componente de alta frequência (AF), com variação de 0,15 a 0,4 Hz, que corresponde à modulação respiratória e é um indicador da modulação do nervo vago sobre o coração (Malliani et al., 1991; Task Force, 1996); componente de baixa frequência (BF), com variação entre 0,04 e 0,15 Hz, que é decorrente da ação conjunta do componente vagal e simpático sobre o coração, sendo o componente simpático predominante (Akselrod et al., 1981; Malliani et al., 1991; Longo et al., 1995; Task Force, 1996; Lombardi et al., 1996; Unalacak et al., 2004; Urakawa \& Yokoyama, 2005; Iwanaga et al., 2005), portanto, essa banda de frequência tem sido utilizada como marcador da modulação simpática atuante no coração (Longo et al., 1995; European Society of Cardiology e the North American Society of Pacing Electrophysiology, 1996); componente de muito baixa frequiência (MBF), com variação entre 0 e 0,04 Hz, cuja explicação fisiológica não está bem definida e parece estar relacionada ao sistema renina-angiotensinaaldosterona, termorregulação e tônus vasomotor periférico (Akselrod et al., 1981; Longo et al., 1995; European Society of Cardiology e the North American Society of Pacing Electrophysiology, 1996).

Alguns estudos têm demonstrado que uma forma de caracterizar o balanço simpato-vagal seria a utilização da razão $\mathrm{BF} / \mathrm{AF}$, que reflete as interações absolutas e relativas entre os componentes simpático e parassimpático do sistema nervoso 
autônomo no coração (Malliani et al., 1991; European Society of Cardiology e the North American Society of Pacing Electrophysiology, 1996). Outra forma de análise seria normalizar os dados para minimizar os efeitos das alterações da banda de MBF. Tal normalização se dá por meio da divisão da potência de um dado componente pela potência total, subtraída do componente de MBF e multiplicada por 100 (Pagani et al., 1988; Malliani et al., 1991; European Society of Cardiology e the North American Society of Pacing Electrophysiology, 1996). O estudo dos efeitos da música sobre a modulação autonômica da frequência cardíaca também vem sendo realizado com aplicação na prática clínica, como por exemplo, associado ao tratamento de câncer (Chuang et al., 2011), na ansiedade pré-operatória (Lee et al., 2011; Lee et al., 2012), em demência ou doenças cerebrovasculares de jovens (Okada et al., 2009), assim como em diferentes faixas etárias (Seiji da Silva et al., 2021), dentre outros. Assim é evidente o efeito da música sobre o sistema nervoso autônomo e consequentemente sobre a variabilidade da frequência cardíaca. No entanto é necessário elucidar se as respostas são diferentes em relação aos diferentes tipos de estímulo musical, bem como a condição do indivíduo estudado em relação ao sexo e se musicalizado ou não. Entretanto, foram escassos os trabalhos encontrados na literatura sobre o estudo do efeito da música sobre a modulação autonômica da frequência cardíaca (Okada et al., 2009; Nakahara et al., 2010; Ellis \&Thayer, 2010), principalmente quando se relaciona à questão dos diferentes tipos de música.

Desta maneira o presente estudo teve como objetivo avaliar a modulação autonômica da frequência cardíaca sob o efeito de estímulo musical em indivíduos musicalizados e não musicalizados e a influência dos diferentes tipos de música e sexo sobre a modulação autonômica da frequência cardíaca. Tendo como hipótese que tanto em indivíduos musicalizados ou não e independente do sexo há alteração da modulação autonômica da frequência cardíaca em indivíduos sob efeito do estímulo musical.

\section{Metodologia}

Estudo experimental prospectivo controlado, cego, desenvolvido no Laboratório de Cardiologia, no Centro Educacional da Universidade Federal do Triângulo Mineiro (UFTM), no município de Uberaba, MG, aprovado pelos Comitês de Ética em Pesquisa da UFTM e da Universidade Federal de São Paulo (UNIFESP), sob parecer da Universidade Federal dos Vales do Jequitinhonha e Mucuri (UFVJM) e validado pela UFTM no 012/09, CAAE: 00848412.0.0000.5505 com registro nas Pró-reitorias de Pesquisa e Pós-Graduação das referidas Universidades.

Todos os voluntários foram informados sobre os procedimentos experimentais aos quais seriam submetidos, do caráter não invasivo dos testes, bem como no fato destes não afetarem sua saúde. Houve esclarecimentos quanto ao sigilo das informações colhidas durante a realização do trabalho, resguardando suas identidades. Os indivíduos que concordaram em participar assinaram o termo de consentimento livre e esclarecido, de acordo com as normas do Conselho Nacional de Saúde (196/96).

Foram estudados 96 voluntários, sendo 44 musicalizados, idade média de 24,6 anos (26 homens e 18 mulheres) e 52 não musicalizados, idade média de 23,9 anos (22 homens e 30 mulheres). Todos os voluntários foram inicialmente triados entre os alunos e funcionários da Universidade Federal do Triângulo Mineiro (UFTM), para comporem o grupo não musicalizado (GNM); e entre estudantes do Conservatório Estadual de Música Renato Frateschi, para compor o grupo musicalizado (GM), ambos situados no Município de Uberaba (MG), por meio de amostra de conveniência.

Os critérios de exclusão foram os voluntários que apresentassem diagnósticos ou relato de distúrbios auditivos, doenças cardiovasculares ou pulmonares, fumantes e os que fizessem uso de medicamentos betabloqueadores, diuréticos, bloqueadores de canal de cálcio e inibidores de enzima conversora de angiotensina, antidepressivos, ou qualquer impedimento pessoal que o indivíduo apresentasse, uma vez que sua participação no estudo foi de caráter voluntário. Não foram incluídos nesse estudo os indivíduos que já haviam realizado terapia de realidade virtual previamente, impossibilitados de permanecer na 
posição ortostática, com comprometimento visual grave ou não compensado com uso de lentes corretivas e crianças com distúrbios ortopédicos que resultavam em quadro álgico. Como critérios de inclusão para o GM foi estabelecido que os voluntários deveriam ser estudantes do Conservatório Estadual de Música Renato Frateschi, estudando algum tipo de instrumento musical há no mínimo um ano, sem interrupção. Para o GNM foi estabelecido que o voluntário deveria ser estudante ou funcionário da UFTM, e que nunca tivesse realizado qualquer tipo de estudo ou treinamento musical.

\subsection{Procedimentos experimentais e controle ambiental}

Os experimentos foram realizados sempre no mesmo período do dia para reduzir as influências das variações circadianas sobre o organismo. Foi recomendado aos voluntários que comparecessem com roupas e calçados confortáveis, que não fizessem uso de bebidas alcoólicas e/ou estimulantes 24 horas antes dos testes, que fizessem uma refeição leve pelo menos duas horas antes do teste e que não realizassem atividade física extenuante no dia anterior.

A preparação dos equipamentos, dos materiais e a organização da sala foram realizadas com uma hora de antecedência à chegada de cada voluntário. Para reduzir a ansiedade e expectativa por parte dos voluntários, foram feitos procedimentos de familiarização dos indivíduos com o protocolo de teste, com a equipe de pesquisadores e equipamentos, no mínimo uma semana antes dos protocolos experimentais. Os protocolos foram realizados sempre pelos mesmos avaliadores, nos dois grupos estudados. Com o mesmo intuito, foi mantido um trânsito mínimo de pessoas no laboratório durante a execução dos experimentos.

\subsection{Protocolo experimental: avaliação da modulação autonômica da frequência cardíaca}

Este teste teve como objetivo avaliar a modulação autonômica da resposta da frequência cardíaca a partir de sua variabilidade (VFC) durante o repouso em silêncio e sob estímulo musical. Foi utilizado um monitor de frequência cardíaca, da marca POLAR, modelo RS800CX para obtenção dos dados de FC e posterior análise da VFC. Primeiramente foi feito o posicionamento da cinta no tórax (na altura do coração) e do relógio no pulso do voluntário, além do fone de ouvido. A partir de então, os voluntários foram orientados a manter-se em repouso na posição sentada, com uma respiração tranquila e evitando conversar com os avaliadores. Após dez minutos, para estabilização das variáveis cardiorrespiratórias, foi iniciada a coleta dos dados de frequência cardíaca de repouso por 5 minutos e trinta segundos, na posição sentada em uma sala com o ambiente silencioso (Seiji et al., 2021). Depois desse período, os voluntários foram expostos a um período de 22 minutos de música, mantendo a mesma coleta de FC. Foram impostas quatro músicas com ritmos diferentes durante cinco minutos e trinta segundos cada uma (Tabela 1).

Tabela 1. Música impostas durante as avaliações.

\begin{tabular}{llc}
\hline Música & \multicolumn{1}{c}{ Nome e estilo musical } & Adquirida \\
\hline 1 & Terceira Sinfonia (Heróica), de Beethoven música clássica, instrumental, ritmo lento. & Via Itunes \\
2 & Day Ligth Daylight of Konoha, de Toshiro Masuda música instrumental, ritmo lento. & Via Itunes \\
3 & Bad Romance, de Lady Gaga música pop, vozes, ritmo rápido. & Via Itunes \\
4 & Drum Solos, de Kitaro música instrumental percussão ritmo lento. & Via Itunes \\
\hline
\end{tabular}

Fonte: Autores.

Após a coleta da FC, foram descartados os 15 segundos iniciais e finais de cada amostra, a fim de selecionar trechos mais estáveis de sinal. Resultando em um tempo total de cinco minutos de análise em cada condição, totalizando 25 minutos por voluntário. Seguido por uma inspeção visual da distribuição dos dados de FC coletados, para verificar a estabilidade do 
traçado obtido, bem como a ausência de artefatos, para a realização das análises no domínio do tempo e da frequência, fornecidos pelo software Polar Precision Performance Sw, que acompanha o monitor de FC.

\subsection{Métodos de análise}

\subsubsection{Análise da resposta da variabilidade da frequência cardíaca no domínio do tempo}

Para a análise dos dados no domínio do tempo foram utilizados os índices RMSSD dos intervalos R-R (iR-R) ((raiz quadrada da média do quadrado das diferenças entre intervalos da variabilidade da frequência cardíaca consecutivos. Este parâmetro fornece uma quantificação das variações abruptas da variabilidade) e pNN50 (NN50: quantidade absoluta (contagem) de intervalos NN que diferem mais de 50ms em relação ao intervalo anterior - pNN50: porcentagem de NN50 em relação à quantidade total de intervalos NN) (Antila, 1979).

\subsubsection{Análise no domínio da frequência}

Por meio da análise espectral foram obtidas as bandas de muito baixa frequência, de baixa frequência (BF), e de alta frequência (AF). Em nosso estudo utilizamos duas faixas de frequência que melhor representam a atuação dos componentes vagal e simpático no controle da $\mathrm{FC}$, ou seja, faixa $\mathrm{BF}$, correspondendo de 0,04 a $0,15 \mathrm{~Hz}$ e a faixa de $\mathrm{AF}$, que corresponde de 0,15 a $0,4 \mathrm{~Hz}$. Além dos valores absolutos de $\mathrm{BF}$ e AF, tais componentes também foram expressos como a razão entre as áreas absolutas de baixa e alta frequência (razão BF/AF), que é indicativo do balanço simpatovagal.

\subsection{Análise estatística}

Após a verificação da distribuição Gaussiana dos dados, foi feito a verificação da normalidade dos dados por meio do teste de Shapiro Wilk. Foram realizadas comparações intragrupos entre as condições de silêncio e os diferentes tipos de música dos valores médios dos índices RMSSD e pNN50 no domínio do tempo. Já no domínio da frequência, foram utilizadas a razão BF/AF, BF e AF. O teste estatístico utilizado foi One-way ANOVA, com post-hoc de Tukey, por meio do software GraphPad Instat, versão 3, 1997. Para a comparação intergrupos, foi utilizado o teste t não pareado comparando os valores médios dos índices RMSSD e pNN50, no domínio do tempo. O mesmo teste foi utilizado para a comparação dos índices no domínio da frequência: AF, BF e a razão BF/AF. A comparação intergrupos foi utilizada para comparar indivíduos musicalizados e não musicalizados, bem como a diferença entre os sexos feminino e masculino. Todos os testes estatísticos utilizados neste estudo tiveram nível de significância estabelecido em 5\%.

\section{Resultados}

Na Tabela 2 estão apresentados os resultados dos índices de VFC no domínio do tempo (RMSSD e pNN50) e no domínio da frequência $(\mathrm{BF}, \mathrm{AF}$ e $\mathrm{BF} / \mathrm{AF})$, referentes às condições de repouso em silêncio e durante exposição a diferentes tipos de música, dos indivíduos musicalizados. Em relação aos índices do domínio do tempo, foi observado valores significativamente menores $(\mathrm{p}<0,05)$ a partir da música 1 em relação ao repouso no grupo feminino, exceto no índice RMSSD na música 3. Já para o grupo masculino, foram observadas diferenças estatisticamente significantes $(\mathrm{p}<0,05)$ a partir da música 2 em relação ao repouso, também identificando valores menores de RMSSD e pNN50 durante a exposição musical. Já em relação aos índices no domínio da frequência, os valores de baixa frequência que indicam a atividade simpática, foram significativamente maiores $(\mathrm{p}<0,05)$ durante a execução da música 3 em relação ao repouso e à música 4 , no grupo feminino. Ainda considerando a análise no domínio da frequência, o índice de alta frequência, que indica a atividade parassimpática e a relação BF/AF que representa o balanço simpato-vagal, apresentaram diferença estatisticamente significante em relação ao repouso, a partir da música 2 ( $<<0,05)$, no grupo masculino. Enquanto os valores de AF reduziram 
durante a exposição musical, os valores da razão aumentaram, indicando um predomínio simpático durante o estímulo musical (Tabela 2).

Na Tabela 3 estão apresentados os resultados dos índices de VFC no domínio do tempo (RMSSD e pNN50) e no domínio da frequência $(\mathrm{BF}, \mathrm{AF}$ e $\mathrm{BF} / \mathrm{AF})$, referentes às condições de repouso em silêncio e durante exposição a diferentes tipos de música, dos indivíduos não musicalizados. Em relação ao índice RMSSD foram encontradas diferenças estatisticamente significantes entre a música 3 e o repouso e entre a música 4 e o repouso e à música 1 , no grupo masculino. No grupo feminino foram encontrados valores significativamente menores de RMSSD durante a exposição das músicas 3 e 4 em relação ao repouso e à exposição da música 1. No grupo masculino foram encontrados valores de pNN50 significativamente menores $(\mathrm{p}<0,05)$ a partir da música 2 em relação ao repouso. No grupo feminino foram encontrados valores significativamente menores do índice pNN50 durante a exposição da música 3 em relação ao repouso e música 1 e durante a exposição da música 4 em relação a música 1. Já os valores da banda $\mathrm{AF}$ foram significativamente menores $(\mathrm{p}<0,05)$ durante a exposição das músicas 2 e 3 em relação a exposição da música 1 , no grupo feminino. Verificando os valores da razão $B F / A F$, houve diferença estatisticamente significante $(p<0,05)$, com maiores valores durante a exposição da música 4 em relação ao repouso, no grupo feminino (Tabela 3). Na tabela 4, podemos observar que ao comparar a variabilidade da $\mathrm{FC}$ entre os indivíduos do sexo feminino musicalizados e não musicalizados foi encontrada diferenças estatisticamente significantes ( $\mathrm{p}<0,05)$ no índice RMSSD durante a execução da música 1 (com maiores valores para o grupo não musicalizado) e no índice de BF durante a execução da música 3 (com valores maiores para o grupo musicalizado) (Tabela 4). Quando se compara os grupos musicalizados com os não musicalizados sem distinção de sexo, observa-se que houve diferença estatisticamente significativa $(\mathrm{p}<0,05)$ no índice $B F$ durante a execução da música 3 , com menores valores para o grupo não musicalizado (Tabela 5). Também foi realizada a comparação da VFC entre os sexos masculino e feminino separadamente nos grupos de indivíduos musicalizados e não musicalizados, conforme valores expressos nas tabelas 6 e 7 . No grupo não musicalizado foi encontrada diferença estatisticamente significante $(\mathrm{p}<0,05)$ na banda $\mathrm{AF}$, durante a execução da música 4, evidenciando maiores valores para o grupo feminino, em relação ao grupo masculino (Tabela 6). Já no grupo musicalizado foram encontradas diferenças estatisticamente significantes $(p<0,05)$ na comparação entre os sexos, quando analisado as variáveis no domínio do tempo e da frequência. No domínio do tempo, os índices RMSSD e pNN50 foram maiores para o grupo masculino durante a exposição à música 4. No domínio da frequência, o grupo masculino apresentou maiores valores da banda BF em comparação ao grupo feminino, durante o repouso e exposição às músicas 2 e 4 (Tabela 7). 


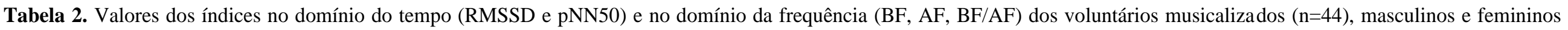
durante repouso e durante a exposição a diferentes tipos de música.

\begin{tabular}{|c|c|c|c|c|c|c|}
\hline VARIÁVEL & & REPOUSO & MÚSICA 1 & MÚSICA 2 & MÚSICA 3 & MÚSICA 4 \\
\hline \multirow{2}{*}{ RMSSD } & Masculino & $37,79 \pm 23,11$ & $34,25 \pm 24,51$ & $33,15 \pm 23^{R}$ & $31,40 \pm 20,25^{\mathrm{R}}$ & $33,85 \pm 22,16^{R}$ \\
\hline & Feminino & $28,99 \pm 13,45$ & $24,60 \pm 13,66^{R}$ & $24,29 \pm 10,65^{\mathrm{R}}$ & $25,04 \pm 10,94$ & $21,91 \pm 8,66^{\mathrm{R}}$ \\
\hline \multirow{2}{*}{ pNN50 } & Masculino & $9,08 \pm 8,5$ & $7,45 \pm 9,17$ & $6,78 \pm 8,67^{\mathrm{R}}$ & $6,37 \pm 7,91^{\mathrm{R}}$ & $7,05 \pm 8,19^{\mathrm{R}}$ \\
\hline & Feminino & $5,70 \pm 5,15$ & $3,64 \pm 4,07^{R}$ & $3,44 \pm 3,29^{R}$ & $3,42 \pm 3,68^{R}$ & $2,15 \pm 1,89^{\mathrm{R}}$ \\
\hline \multirow{2}{*}{$\mathrm{BF}$} & Masculino & $28,07 \pm 8,28$ & $29,18 \pm 10,15$ & $29,84 \pm 9,97$ & $29,42 \pm 8,00$ & $26,99 \pm 6,67$ \\
\hline & Feminino & $22,54 \pm 9,44$ & $25,92 \pm 7,90$ & $25,36 \pm 6,50$ & $30,15 \pm 5,84^{\mathrm{R}, 4^{*}}$ & $23,72 \pm 5,78$ \\
\hline \multirow{2}{*}{$\mathrm{AF}$} & Masculino & $18,12 \pm 10,29$ & $16,08 \pm 12,01$ & $14,42 \pm 10,72^{R}$ & $12,55 \pm 8,74^{R}$ & $13,55 \pm 8,82^{R}$ \\
\hline & Feminino & $16,98 \pm 10,87$ & $18,41 \pm 13,38$ & $13,09 \pm 6,74$ & $14,52 \pm 6,94$ & $14,75 \pm 8,42$ \\
\hline \multirow{2}{*}{$\mathrm{BF} / \mathrm{AF}$} & Masculino & $2,14 \pm 1,29$ & $3,31 \pm 3,20$ & $3,71 \pm 3,26^{\mathrm{R}}$ & $3,59 \pm 2,97^{\mathrm{R}}$ & $3,79 \pm 3,6^{\mathrm{R}}$ \\
\hline & Feminino & $2,46 \pm 3,23$ & $2,35 \pm 1,72$ & $2,38 \pm 1,36$ & $2,50 \pm 1,11$ & $2,50 \pm 2,73$ \\
\hline
\end{tabular}

${ }^{\mathrm{R}}$ Diferença significativa em relação ao Repouso. ${ }^{4 *}$ Diferença significativa em relação à música 4 . Nível de significância $\mathrm{p}<0,05$.

Fonte: Autores. 


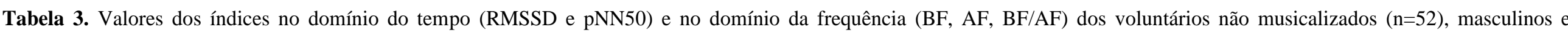
femininos durante repouso e durante a exposição a diferentes tipos de música.

\begin{tabular}{|c|c|c|c|c|c|c|}
\hline VARIÁVEL & & REPOUSO & MÚSICA 1 & MÚSICA 2 & MÚSICA 3 & MÚSICA 4 \\
\hline \multirow{2}{*}{ RMSSD } & Masculino & $39,41 \pm 20,30$ & $36,68 \pm 20,62$ & $35,48 \pm 22,61$ & $33,60 \pm 20,25^{\mathrm{R}}$ & $31,84 \pm 17,82^{\mathrm{R}, 1^{*}}$ \\
\hline & Feminino & $39,13 \pm 25,35$ & $39,06 \pm 27,04$ & $37,01 \pm 26,65$ & $34,57 \pm 25,45^{\mathrm{R}, 1^{*}}$ & $34,40 \pm 26,01^{\mathrm{R}, 1^{*}}$ \\
\hline \multirow{2}{*}{ pNN50 } & Masculino & $9,02 \pm 7,70$ & $7,61 \pm 7,92$ & $6,80 \pm 7,80^{R}$ & $6,73 \pm 8,42^{\mathrm{R}}$ & $5,82 \pm 6,76^{\mathrm{R}}$ \\
\hline & Feminino & $8,40 \pm 9,69$ & $8,61 \pm 10,70$ & $7,72 \pm 9,99$ & $6,56 \pm 8,91^{\mathrm{R}, 1^{*}}$ & $6,87 \pm 9,86^{1 *}$ \\
\hline \multirow{2}{*}{$\mathrm{BF}$} & Masculino & $25,86 \pm 8,65$ & $26,55 \pm 8,28$ & $27,06 \pm 10,65$ & $27,77 \pm 10,25$ & $27,52 \pm 11,20$ \\
\hline & Feminino & $24,96 \pm 8,91$ & $24,50 \pm 9,23$ & $24,56 \pm 10,43$ & $23,44 \pm 7,70$ & $23,82 \pm 7,88$ \\
\hline \multirow{2}{*}{$\mathrm{AF}$} & Masculino & $15,60 \pm 10,55$ & $15,40 \pm 9,10$ & $12,79 \pm 6,12$ & $12,24 \pm 7,93$ & $10,63 \pm 5,79$ \\
\hline & Feminino & $18,62 \pm 10,02$ & $20,15 \pm 12,15$ & $15,05 \pm 11,13^{1 *}$ & $15,01 \pm 9,16^{1^{*}}$ & $16,32 \pm 11,38$ \\
\hline \multirow{2}{*}{$\mathrm{BF} / \mathrm{AF}$} & Masculino & $2,65 \pm 2,30$ & $2,58 \pm 1,83$ & $2,68 \pm 1,77$ & $3,12 \pm 1,96$ & $3,36 \pm 2,12$ \\
\hline & Feminino & $1,85 \pm 1,37$ & $1,89 \pm 1,49$ & $2,44 \pm 1,97$ & $2,48 \pm 2,47$ & $2,57 \pm 2,69^{\mathrm{R}}$ \\
\hline
\end{tabular}

${ }^{\mathrm{R}}$ Diferença significativa em relação ao Repouso. ${ }^{1 *}$ Diferença significativa em relação à música 1. Nível de significância p<0.05. Fonte: Autores. 
Tabela 4. Valores dos índices da VFC no domínio do tempo (RMSSD e pNN50) e no domínio da frequência (BF, AF, BF/AF) dos voluntários do grupo feminino, musicalizados $(n=18)$ e não musicalizados $(n=30)$, durante repouso e durante a exposição a diferentes tipos de música.

\begin{tabular}{|c|c|c|c|c|c|c|}
\hline VARIÁVEL & & REPOUSO & MÚSICA 1 & MÚSICA 2 & MÚSICA 3 & MÚSICA 4 \\
\hline \multirow{2}{*}{ RMSSD } & Musicalizados & $28,99 \pm 13,45$ & $24,60 \pm 13,66$ & $24,29 \pm 10,65$ & $25,04 \pm 10,94$ & $21,91 \pm 8,66$ \\
\hline & Não Musicalizados & $39,13 \pm 25,35$ & $39,06 \pm 27,04 *$ & $37,01 \pm 26,65$ & $34,57 \pm 25,45$ & $34,40 \pm 26,01$ \\
\hline \multirow{2}{*}{ pNN50 } & Musicalizados & $5,70 \pm 5,15$ & $3,64 \pm 4,07$ & $3,44 \pm 3,29$ & $3,42 \pm 3,68$ & $2,15 \pm 1,89$ \\
\hline & Não Musicalizados & $8,40 \pm 9,69$ & $8,61 \pm 10,70$ & $7,72 \pm 9,99$ & $6,56 \pm 8,91$ & $6,87 \pm 9,86$ \\
\hline \multirow{2}{*}{$\mathrm{BF}$} & Musicalizados & $22,54 \pm 9,44$ & $25,92 \pm 7,90$ & $25,36 \pm 6,50$ & $30,15 \pm 5,84$ & $23,72 \pm 5,78$ \\
\hline & Não Musicalizados & $24,96 \pm 8,91$ & $24,50 \pm 9,23$ & $24,56 \pm 10,43$ & $23,44 \pm 7,70^{*}$ & $23,82 \pm 7,88$ \\
\hline \multirow{2}{*}{$\mathrm{AF}$} & Musicalizados & $16,98 \pm 10,87$ & $18,41 \pm 13,38$ & $13,09 \pm 6,74$ & $14,52 \pm 6,94$ & $14,75 \pm 8,42$ \\
\hline & Não Musicalizados & $18,62 \pm 10,02$ & $20,15 \pm 12,15$ & $15,05 \pm 11,13$ & $15,01 \pm 9,16$ & $16,32 \pm 11,38$ \\
\hline \multirow{2}{*}{$\mathrm{BF} / \mathrm{AF}$} & Musicalizados & $2,46 \pm 3,23$ & $2,35 \pm 1,72$ & $2,38 \pm 1,36$ & $2,50 \pm 1,11$ & $2,50 \pm 2,73$ \\
\hline & Não Musicalizados & $1,85 \pm 1,37$ & $1,89 \pm 1,49$ & $2,44 \pm 1,97$ & $2,48 \pm 2,47$ & $2,57 \pm 2,69$ \\
\hline
\end{tabular}

* Diferença significativa em relação ao grupo musicalizado. Nível de significância p<0,05. Fonte: Autores. 


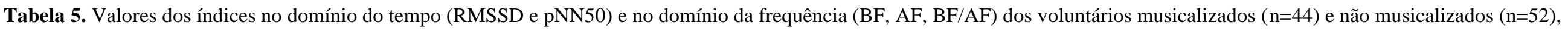
durante repouso e durante a exposição a diferentes tipos de música.

\begin{tabular}{|c|c|c|c|c|c|c|}
\hline VARIÁVEL & & REPOUSO & MÚSICA 1 & MÚSICA 2 & MÚSICA 3 & MÚSICA 4 \\
\hline \multirow{2}{*}{ RMSSD } & Musicalizados & $34,2 \pm 20,0$ & $30,3 \pm 21,1$ & $29,5 \pm 19,3$ & $28,8 \pm 17,2$ & $29,0 \pm 18,7$ \\
\hline & Não Musicalizados & $39,3 \pm 23,1$ & $38,1 \pm 24,3$ & $36,4 \pm 24,8$ & $34,2 \pm 23,2$ & $33,3 \pm 22,7$ \\
\hline \multirow{2}{*}{ pNN50 } & Musicalizados & $7,7 \pm 7,4$ & $5,9 \pm 7,7$ & $5,4 \pm 7,1$ & $5,2 \pm 6,6$ & $5,0 \pm 6,8$ \\
\hline & Não Musicalizados & $8,7 \pm 8,8$ & $8,2 \pm 9,5$ & $7,3 \pm 9,1$ & $6,6 \pm 8,6$ & $6,4 \pm 8,6$ \\
\hline \multirow{3}{*}{$\mathrm{BF}$} & Musicalizados & $25,8 \pm 9,1$ & $27,8 \pm 9,3$ & $28,0 \pm 8,9$ & $29,7 \pm 7,1$ & $25,7 \pm 6,5$ \\
\hline & & & & & & \\
\hline & Não Musicalizados & $25,3 \pm 8,7$ & $25,4 \pm 8,8$ & $25,6 \pm 10,5$ & $25,3 \pm 9,0 *$ & $25,4 \pm 9,5$ \\
\hline \multirow{2}{*}{$\mathrm{AF}$} & Musicalizados & $17,7 \pm 10,4$ & $17,0 \pm 12,5$ & $13,9 \pm 9,2$ & $13,4 \pm 8,0$ & $14,0 \pm 8,6$ \\
\hline & Não Musicalizados & $17,3 \pm 10,3$ & $18,1 \pm 11,1$ & $14,1 \pm 9,3$ & $13,8 \pm 8,7$ & $13,9 \pm 9,8$ \\
\hline \multirow{2}{*}{$\mathrm{LF} / \mathrm{HF}$} & Musicalizados & $2,3 \pm 2,3$ & $2,9 \pm 2,7$ & $3,2 \pm 2,7$ & $3,1 \pm 2,4$ & $3,0 \pm 3,0$ \\
\hline & Não Musicalizados & $2,2 \pm 1,8$ & $2,2 \pm 1,7$ & $2,5 \pm 1,9$ & $2,8 \pm 2,3$ & $2,9 \pm 2,5$ \\
\hline
\end{tabular}

* Diferença significativa em relação ao grupo musicalizado. Nível de significância $p<0,05$.

Fonte: Autores. 
Tabela 6. Valores dos índices no domínio do tempo (RMSSD e pNN50) e no domínio da frequência (BF, AF, BF/AF) dos voluntários não musicalizados $(\mathrm{n}=44)$, subdivididos em grupos masculino e feminino.

\begin{tabular}{|c|c|c|c|c|c|c|}
\hline VARIÁVEL & & REPOUSO & MÚSICA 1 & MÚSICA 2 & MÚSICA 3 & MÚSICA 4 \\
\hline \multirow[b]{2}{*}{ RMSSD } & Feminino & $34,7 \pm 19,0$ & $34,2 \pm 19,4$ & $31,7 \pm 18,4$ & $29,2 \pm 17,5$ & $28,4 \pm 16,4$ \\
\hline & Masculino & $39,4 \pm 20,3$ & $36,7 \pm 20,6$ & $35,5 \pm 22,6$ & $33,6 \pm 20,2$ & $31,8 \pm 17,8$ \\
\hline \multirow{2}{*}{ pNN50 } & Feminino & $6,8 \pm 7,7$ & $6,7 \pm 7,8$ & $5.8 \pm 7.5$ & $4.9 \pm 6.9$ & $4.5 \pm 6.2$ \\
\hline & Masculino & $9,0 \pm 7,7$ & $7,6 \pm 7,9$ & $6.8 \pm 7.8$ & $6.7 \pm 8.4$ & $5.8 \pm 6.8$ \\
\hline \multirow[b]{2}{*}{$\mathrm{BF}$} & Feminino & $25.0 \pm 8.9$ & $24.5 \pm 9.2$ & $24.6 \pm 10.4$ & $23.4 \pm 7.7$ & $23.8 \pm 7.9$ \\
\hline & Masculino & $25.9 \pm 8.6$ & $26.6 \pm 8.3$ & $27.1 \pm 10.7$ & $27.8 \pm 10.2$ & $27.5 \pm 11.2$ \\
\hline \multirow{2}{*}{$\mathrm{AF}$} & Feminino & $18.6 \pm 10.0$ & $20.1 \pm 12.1$ & $15.0 \pm 11.1$ & $15.0 \pm 9.2$ & $16.3 \pm 11.4^{*}$ \\
\hline & Masculino & $15.6 \pm 10.6$ & $15.4 \pm 9.1$ & $12.8 \pm 6.1$ & $12.2 \pm 7.9$ & $10.6 \pm 5.8$ \\
\hline \multirow{2}{*}{$\mathrm{LF} / \mathrm{HF}$} & Feminino & $1.9 \pm 1.4$ & $1.9 \pm 1.5$ & $2.4 \pm 2.0$ & $2.5 \pm 2.5$ & $2.6 \pm 2.7$ \\
\hline & Masculino & $2.7 \pm 2.3$ & $2.6 \pm 1.8$ & $2.7 \pm 1.80$ & $3.1 \pm 2.0$ & $3.4 \pm 2.1$ \\
\hline
\end{tabular}

* Diferença significativa em relação aos sexos. Nível de significância p<0,05.

Fonte: Autores. 


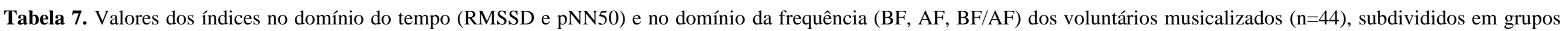
masculino e feminino.

\begin{tabular}{|c|c|c|c|c|c|c|}
\hline VARIÁVEL & & REPOUSO & MÚSICA 1 & MÚSICA 2 & MÚSICA 3 & MÚSICA 4 \\
\hline \multirow{2}{*}{ RMSSD } & Feminino & $29.0 \pm 13.5$ & $24.6 \pm 13.7$ & $24.3 \pm 10.7$ & $25.0 \pm 10.9$ & $21.9 \pm 8.7 *$ \\
\hline & Masculino & $37.8 \pm 23.1$ & $34.3 \pm 24.5$ & $33.2 \pm 23.0$ & $31.4 \pm 20.2$ & $33.8 \pm 22.2$ \\
\hline \multirow{2}{*}{ pNN50 } & Feminino & $5.7 \pm 5.1$ & $3.6 \pm 4.1$ & $3.4 \pm 3.3$ & $3.4 \pm 3.7$ & $2.2 \pm 1.9 *$ \\
\hline & Masculino & $9.1 \pm 8.5$ & $7.5 \pm 9.2$ & $6.8 \pm 8.7$ & $6.4 \pm 7.9$ & $7.0 \pm 8.2$ \\
\hline \multirow{2}{*}{$\mathrm{BF}$} & Feminino & $22.5 \pm 9.4^{*}$ & $25.9 \pm 7.9$ & $25.4 \pm 6.5^{*}$ & $30.2 \pm 5.8$ & $23.7 \pm 5.8^{*}$ \\
\hline & Masculino & $28.1 \pm 8.3$ & $29.2 \pm 10.1$ & $29.8 \pm 10.0$ & $29.4 \pm 8.0$ & $27.0 \pm 6.7$ \\
\hline \multirow{2}{*}{$\mathrm{AF}$} & Feminino & $17.0 \pm 10.9$ & $18.4 \pm 13.4$ & $13.1 \pm 6.7$ & $14.5 \pm 6.9$ & $14.8 \pm 8.4$ \\
\hline & Masculino & $18.1 \pm 10.3$ & $16.1 \pm 12.0$ & $14.4 \pm 10.7$ & $12.5 \pm 8.7$ & $13.5 \pm 8.8$ \\
\hline \multirow{2}{*}{ LF/HF } & Feminino & $2.5 \pm 3.2$ & $2.4 \pm 1.7$ & $2.4 \pm 1.4$ & $2.5 \pm 1.1$ & $2.5 \pm 2.7$ \\
\hline & Masculino & $2.1 \pm 1.3$ & $3.3 \pm 3.2$ & $3.7 \pm 3.3$ & $3.6 \pm 3.0$ & $3.4 \pm 3.2$ \\
\hline
\end{tabular}

* Diferença significativa em relação aos sexos. Nível de significância $p<0,05$.

Fonte: Autores. 


\section{Discussão}

Os efeitos da música sobre a fisiologia tem despertado o interesse de pesquisadores que estudam vários aspectos incluindo respostas cardiorrespiratórias, humorais, autonômicas entre outras, tanto de forma aguda (efeito imediato da música sobre esses sistemas), quanto de forma crônica, como por exemplo avaliando os efeitos terapêuticos do tratamento musical sobre diferentes tipos de doenças ou síndromes. Procuramos estudar o efeito da música sobre o sistema nervoso autônomo e consequentemente sobre a variabilidade da frequência cardíaca, comparando as respostas em relação aos diferentes tipos de estímulo musical, em indivíduos musicalizados e não musicalizados, de ambos os sexos, por serem escassos e por vezes contraditórios, na bibliografia consultada. Buscou-se neste estudo a diversificação da amostra tanto na inserção de homens e mulheres em ambos os grupos, como padronização da coleta de dados. No presente trabalho, foi utilizado a VFC para avaliar a função autonômica cardíaca, por se tratar de um método não-invasivo, eficaz e de baixo custo.

Assim, considerando-se que o presente estudo realizou registros eletrocardiográficos de curta duração, optamos por analisar somente as bandas de BF e AF, que refletem melhor a atuação dos sistemas simpático e parassimpático sobre o coração, bem como a razão entre elas (BF/AF) que reflete o balanço simpato-vagal. Além disso, foram escassos os trabalhos que fizeram esse tipo de avaliação durante a exposição à estímulos musicais, principalmente quando se considera diferentes tipos de música. As músicas selecionadas representaram um espectro variado e foram selecionadas de acordo com características de ritmo, complexidade, timbre, intensidade diversos, procurando-se diferenciá-las quanto aos aspectos excitativo (M3) e calmo ou sedativo (M1 e M2) e intermediário (M4).

No presente estudo foram observadas variações em relação aos componentes da VFC, tanto no domínio do tempo quanto no domínio da frequência quando analisadas durante o repouso em silêncio e durante a exposição aos diferentes tipos de música. No grupo musicalizado feminino foram observados menores valores dos índices do domínio do tempo (RMSSD e pNN50) a partir da música 1 em relação ao repouso, com exceção do RMSSD na música 3. Esse resultado mostra que houve uma redução da atividade parassimpática durante o ato de ouvir música, porém sem diferença entre as músicas. Já em relação ao grupo masculino, houve alterações na modulação autonômica a partir da música $2 \mathrm{em}$ relação ao repouso, sendo que a música clássica não chegou a gerar alterações significativas nesse grupo. Da mesma forma que no grupo feminino não houve diferença entre as músicas 2,3 e 4 . Ou seja, independente do sexo, houve menor atuação parassimpática durante exposição aos diferentes tipos de música.

Em relação aos resultados no domínio do tempo, os dados do presente estudo são discordantes do estudo de Iwanaga \& Moroki (1999) que encontraram diferença na resposta entre música excitativa e sedativa. Porém esses autores não fizeram a comparação com a condição de repouso em silêncio. Ainda, foram avaliadas as respostas fisiológicas (frequência cardíaca, frequência respiratória e pressão arterial) e não diretamente os índices da VFC.

Chuang et al., 2011, encontraram que o RMSSD aumenta durante o tratamento musical, porém o desenho metodológico também diferiu do presente estudo, já que esses autores estudaram pacientes diagnosticados com câncer e o tratamento musical constou de oito sessões de terapia musical com duração de duas horas cada uma.

Já, em relação à análise no domínio da frequência, no grupo feminino houve alteração no índice BF, que mostrou maiores valores quando comparado às condições de repouso e de exposição à música 4 , indicando predomínio simpático durante a execução da música 3, que é considerada uma música de ritmo alto, mais agitada. Por outro lado, no grupo masculino, houve alteração do componente $\mathrm{AF}$, que representa o componente parassimpático, a partir da música 2 em relação ao repouso. Tal redução, ainda, levou a modificação do balanço simpato-vagal, avaliado por meio da razão BF/AF, que aumentou durante a exposição às músicas 2,3 e 4, em relação ao repouso. Os resultados do presente estudo são concordantes com o trabalho de Iwanaga et al. (2005), que encontraram aumento da banda de BF e da razão BF/AF tanto durante a execução de música excitativa quanto sedativa. 
Em relação a banda de AF, esta não diferiu durante a exposição a música clássica (sedativa) em relação ao repouso em silêncio, mostrando até pequeno aumento sem significância estatística no grupo feminino. Tal resultado também é concordante com o estudo de Iwanaga et al.(2005) que relataram valores maiores da banda de AF durante a exposição à música sedativa quando comparada a excitativa.

Em relação ao grupo não musicalizado, também foram encontradas variações nos índices da VFC, tanto no domínio do tempo, quanto no domínio da frequência.

Em relação ao índice RMSSD, no grupo masculino foi observado menores valores deste índice durante a exposição à música 3 em relação à condição de repouso e durante a exposição à música 4 em relação ao repouso e à música 1 . Já no grupo feminino foi observado redução dos valores de RMSSD durante as músicas 3 e 4 em relação ao repouso e música 1 . Tal resultado identifica uma redução progressiva do tônus parassimpático na transição do silêncio para exposição a estímulo musicais, porém encontrando significância significativa apenas a partir da música 3 , considerada uma música excitativa. $\mathrm{O}$ índice pNN50 teve um padrão similar ao anterior, porém foi encontrado redução estatisticamente significativa no grupo masculino a partir da música 2 em relação ao repouso e no grupo feminino na música 3 em relação ao repouso e música 1 e durante a música 4 em relação à música 1 . O padrão de comportamento foi similar entre os grupos musicalizados e não musicalizados, mostrando uma redução progressiva dos índices no domínio do tempo da VFC. Porém no grupo musicalizado a diferença estatística também ocorreu entre os tipos de música, evidenciando uma resposta aumentada ao estímulo musical.Tais resultados sugerem que em indivíduos musicalizados, as diferenças de ritmos musicais não causam tanta influência no sistema nervoso autônomo, pelo treinamento que estes possuem.

Já, em relação aos índices no domínio da frequência, foi observado diferença estatísticamente significativa apenas na banda de alta frequência, que é representativa do componente parassimpático, no grupo feminino, durante as músicas 2 e $3 \mathrm{em}$ relação à música 1 , com redução destes valores, indicando redução do tônus parassimpático durante as músicas de ritmo mais elevado. Já, em relação ao balanço simpato-vagal, foi encontrado valores significativamente maiores da razão BF/AF, grupo feminino, somente durante a música 4 em relação ao repouso. Os resultados do presente estudo são discordantes com Chuang et al (2011) que encontraram aumento das bandas BF e AF durante o tratamento musical e não encontraram diferença estatisticamente significativa da razão entre as bandas. Porém são concordantes com os resultados de Iwanaga et al. (2005) que encontraram que a banda de $\mathrm{AF}$ foi maior na música sedativa quando comparada à excitativa. Corroborando com nossos achados.

Quando foi feita a comparação entre indivíduos musicalizados e não musicalizados, não foram encontradas diferenças estatisticamente significativas entre os grupos, exceto na banda de baixa frequência durante a execução da música 3 onde os indivíduos não musicalizados apresentaram valores menores do que os musicalizados. É esperado, segundo a literatura (Iwanaga et al., 2005), que em músicas consideradas excitativas, como a música 3 utilizada no presente estudo, ocorra um predomínio do componente simpático do sistema nervoso autônomo.

Observando a evolução do grupo musicalizado e não musicalizado durante a execução das músicas observa-se que o grupo musicalizado foi respondendo aos diferentes tipos de música com aumento da banda BF até a música 3, enquanto que no grupo não musicalizado praticamente não houve alteração desta banda, gerando então durante a execução da música 3 , uma diferença significativa entre os grupos.

\subsection{Limitações}

Como limitações do estudo destacamos o número de avaliações de exposições às musicas. 


\section{Conclusão}

Concluímos que ocorre redução do tônus parassimpático na transição do repouso para exposição musical, tanto na análise no domínio do tempo, quanto no domínio da frequência. O padrão de comportamento da VFC em indivíduos musicalizados e não musicalizados é similar, com redução progressiva dos índices no DT. No grupo musicalizado houve diferença nos índices da VFC entre as músicas, inferindo que nesse grupo a resposta esteja aumentada ao estímulo musical quando comparado ao grupo não musicalizado. A modulação autonômica em repouso e durante exposição das músicas 1 , 2 e 3 se mostrou similar entre os grupos feminino e masculino, apresentando diferença na música 4, com maior predomínio parassimpático no sexo masculino. Para trabalhos futuros sugere-se que os resultados alcançados sejam acompanhado ao longo do tempo, em situações de estresse e em diferentes doenças.

\section{Agradecimentos}

Aos servidores da disciplina de Anatomia Humana da UFTM; aos professores e alunos do Conservatório Estadual de Música Renato Frateschi, por todo o apoio ao projeto e a FUNEPU, pelo apoio financeiro.

\section{Referências}

Akselrod, S., Gordon, D., Ubel, F. A., Shannon, D. C., Berger, A. C., \& Cohen, R. J. (1981). Power spectrum analysis of heart rate fluctuation: a quantitative probe of beat-to-beat cardiovascular control. Science. 213(4504):220-2.

Antila K. Quantitative characterization of heart rate during exercise. Scand J Clin Lab Invest Suppl. 1979; (153):3-68.

Blair, S. N., Painter, P., Pate, R. R., Smith, L. K., \& Taylor, C. B. (1988). Resource Manual for Guidelines for Exercise Testing and Prescription. American College of Sports Medicine. Philadelphia, Lea \& Febiger.

Catai, A. M., Chacon-Mikahil, M. P., Martinelli, F. S., Forti, V. A., Silva, E., Golfetti, R., Martins, L. E., Szrajer, J. S., Wanderley, J. S., Lima-Filho, E. C., Milan, L. A., Marin-Neto, J. A., Maciel, B. C., \& Gallo-Junior, L. (2002). Effects of aerobic exercise training on heart rate variability during wakefulness and sleep and cardiorespiratory responses of young and middle-aged healthy men. Braz J Med Biol Res. 35(6): 741-52.

Chuang, C. Y., Han, W. R., Li, P. C., Song, M. Y., \& Young, S. T. (2011). Effect of long-term music therapy intervention on autonomic function in anthracycline-treated breast cancer patients. Integr Cancer Ther. 10(4):312-6.

Ellis, R. J \& Thayer, J. F. (2010). Music and Autonomic Nervous System (Dys)function. Music Percept. 27(4):317-326.

Franchini, K. G. Função e disfunção autonômica na doença cardiovascular. Rev Soc Cardiol Estado de São Paulo. 1998;2:285-297.

Fukui, H \& Toyoshima, K. (2008). Music facilitate the neurogenesis, regeneration and repair of neurons. Med Hypotheses. 71(5):765-9.

Gallo, L. Jr., Maciel, B. C., Marin-Neto, J. A, Martins, L. E., Lima-Filho, E. C., Golfetti, R., Chacon, M. P., \& Forti, V. A. (1995). Control of heart rate during exercise in health and disease. Braz J Med Biol Res. 28(11-12):1179-84.

Iwanaga, M., Kobayashi, A., \& Kawasaki C. (2005). Heart rate variability repetitive exposure to music. Biological Psychology. 70:61-66.

Iwanaga, M \& Moroki, Y. (1999). Subjective and Physiological Responses to Music Stimuli Controlled Over Activity and Preference. J Music Ther. 36(1):2638.

Lee, K. C., Chao, Y. H., Yiin, J. J., Chiang, P. Y., \& Chao, Y. F. (2011). Effectiveness of different music-playing devices for reducing preoperative anxiety: a clinical control study. Int J Nurs Stud. 48(10):1180-7.

Lee, K. C., Chao, Y. H., Yiin, J. J., Hsieh, H. Y., Dai, W. J., \& Chao, Y. F. (2012). Evidence that music listening reduces preoperative patients' anxiety. Biol Res Nurs. 14(1):78-84.

Limb, C. J. (2006). Structural and functional neural correlates of music perception. Anat Rec A Discov Mol Cell Evol Biol. 288(4):435-46.

Lombardi, F., Malliani, A., Pagani, M., \& Cerutti, S. (1996). Heart rate variability and its sympatho-vagal modulation. Cardiovasc Res. 32(2):208-16.

Longo, A., Ferreira, D., \& Correia, M. J. (1995). Variabilidade da freqüencia cardíaca. Ver Port Cardiol.14 (3): $241-62$.

Malliani, A., Pagani, M., Lombardi, F., \& Cerutti, S. (1991). Cardiovascular neural regulation explored in the frequency domain. Circulation. 84(2):482-92.

Melo, G. A. A., Rodrigues, A. B., Firmeza, M. A., Grangeiro, A. S., Oliveira, P. P., \& Caetano, J. A. (2018).Musical intervention on anxiety and vital parameters of chronic renal patients: a randomized clinical trial. Rev. Latino-Am. Enfermagem. 26:e2978. http://dx.doi.org/10.1590/1518-8345.2123.2978 
Nakahara, H., Furuya, S., Francis, P. R., \& Kinoshita, H. (2010). Psycho-physiological responses to expressive piano performance. Int J Psychophysiol. 75(3):268-76.

Nakahara, H., Furuya, S., Masuko, T., Francis, P. R., \& Kinoshita, H. (2011). Performing music can induce greater modulation of emotion-related psychophysiological responses than listening to music. Int J Psychophysiol. 81(3):152-8.

Nieto-Romero, R. M. (2017). Efectos de la musicoterapia sobre el nivel de ansiedad del adulto cardiópata sometido a resonancia magnética. Enfermería Universitaria. 14(2):88-96.

Nogueira, M. L., Garner, D. M., Osório, E., de Abreu, L. C., \& Vitor Engrácia Valenti, V. E. (2015). Globally chaotic analysis of Heart Rate Variability during acute auditory stimulus by heavy metal music . MedicalExpress . 2(5):M150504.

Okada, K., Kurita, A., Takase, B., Otsuka, T., Kodani, E., Kusama, Y., Atarashi, H., \& Mizuno, K. (2009). Effects of music therapy on autonomic nervous system activity, incidence of heart failure events, and plasma cytokine and catecholamine levels in elderly patients with cerebrovascular disease and dementia. Int Heart J. 50(1):95-110

Pagani, M., Malfatto, G., Pierini, S., Casati, R., Masu, A.M., Poli, M., Guzzetti, S., Lombardi, F., Cerutti, S., \& Malliani, A. (1988). Spectral analysis of heart rate variability in the assessment of autonomic diabetic neuropathy. J Auton Nerv Syst. 23(2):143-53.

Peretz, I. (2002). Brain specialization for music. Neuroscientist. 8(4):372-80.

Riganello, F., Candelieri, A., Quintieri, M., Conforti, D., \& Dolce, G. (2010). Heart rate variability: an index of brain processing in vegetative state? An artificial intelligence, data mining study. Clin Neurophysiol. 121(12):2024-34.

Seiji , F., Novais, L. D., Custódio Júnior, J. da Silva., Henriques Júnior, S. Q., Espindula, A. P., \& Smith, R. L. (2021). Impact of music on autonomic modulation in different age groups. Journal Research, Society and Development. 10(8): e1310817030. DOI: 10.33448/rsd-v10i8.17030.

Silva, C. S. da., Marques, L. S., Moraes, F. R., Catai, A. M., \& Oliveira, L. (2001). Investigação da variabilidade da frequência cardíaca de mulheres nos períodos manhã e noite. Rev. Bras. Fisioter. 5(2): 65-71.

Silva, K. F., Felix, M. M., Cruz, L. F., Barichello, E., Pires, P. S., Mattia, A. L., Haas, V. J., \& Barbosa, M. H. (2021). Efeitos da música na ansiedade de doadores de sangue: ensaio clínico randomizado. Acta Paul Enferm. 34:eAPE00461.

Sokhadze, E. M. (2007). Effects of music on the recovery of autonomic and electrocortical activity after stress induced by aversive visual stimuli. Appl Psychophysiol Biofeedback. 32(1):31-50.

Steiner, 1. (1999).Processamento Auditivo Central. Monografia (Especialização em Audiologia Clínica). Centro de Especialização em Fonoaudiologia Clínica-CEFAC.

Task Force. (1996). Heart rate variability: standards of measurement, physiological interpretation and clinical use. Task Force of the European Society of Cardiology and the North American Society of Pacing and Eletrophysiology. Circulation. 93(5):1043-65.

Tshiswaka, S. K., \& \& Pinheiro, S. L. (2020). Effect of music on reducing anxiety in children during dental treatment. RGO, Rev Gaúch Odontol. 68: e20200033. http://dx.doi.org/10.1590/1981-863720200003320190049

Unalacak, M., Aydin, M., Ermis, B., Ozeren, A., Sogut, A., Demirel, F., \& Unluoglu, I. (2004). Assessment of cardiac autonomic regulation in children with monosymptomatic nocturnal enuresis by analysis of heart rate variability. Tohoku J Exp Med. 204(1):63-9.

Urakawa, K., \& Yokoyama, K. (2005). Music can enhance exercise-induced sympathetic dominancy assessed by heart rate variability. Tohoku J Exp Med. 206(3):213-8.

Zatorre, R. J., \& Salimpoor, V. N. (2013). From perception to pleasure: music and its neural substrates. Proc Natl Acad Sci U S A. 2:10430-7. 